\title{
Kohonen Self-Organizing Map for the Traveling Salesperson Problem
}

\author{
Lucas Brocki \\ Polish-Japanese Institute of Information Technology, \\ ul. Koszykowa 86, 02-008 Warsaw, Poland \\ lukasz.brocki@pjwstk.edu.pl
}

\begin{abstract}
This work shows how a modified Kohonen Self-Organizing Map with one dimensional neighborhood is used to approach the symmetrical Traveling Salesperson Problem (TSP). Solution generated by the Kohonen network is improved by the 2opt algorithm. The paper describes briefly self-organization in neural networks, 2opt algorithm and modifications applied to Self-Organizing Map. Finally, the algorithm is compared with Evolutionary Algorithm with Enhanced Edge Recombination operator and Lin-Kerninghan algorithm.
\end{abstract}

\section{Kohonen Self-Organizing Map basics}

In 1975 Teuvo Kohonen introduced new type of neural network that uses competitive, unsupervised learning [1]. This approach is based on WTA (Winner Takes All) and WTM (Winner Takes Most) algorithms. Therefore, these algorithms will explained here briefly. The most basic competitive learning algorithm is WTA. When input vector (a pattern) is presented, a distance to each neuron's synaptic weights is calculated. The neuron whose weights are most correlated to current input vector is the winner. Correlation is equal to scalar product of input vector and considered synaptic weights. Only the winning neuron modifies it's synaptic weights to the point presented by input pattern. Synaptic weights of other neurons do not change. The learning process can be described by the following equation:

$$
W_{i} \leftarrow W_{i}+\eta\left(x-W_{i}\right)
$$

where $i \in[0 .$. numer of neurons $], W_{i}$ represents all synaptic weights of the winning neuron, $\eta$ is learning rate and $x$ stands for current input vector. This simple algorithm can be extended. The most common extension is giving more chance of winning to neurons that are rarely activated. However, WTM strategy has better convergence than WTA. The difference between those two algorithms is that many neurons in WTM strategy adapt their synaptic weights in one learning iteration. In this case not only the winner, but also it's neighborhood adapts. The farther is the neighboring neuron from the winner, the smaller modification is applied to it's weights. This adaptation process can be described as:

$$
W_{i} \leftarrow W_{i}+\eta N(i, x)\left(x-W_{i}\right)
$$

for all neurons $i$ that belong to winner's neighborhood. $W_{i}$ stands for synaptic weights of neuron $i$ and $x$ is current input vector. $\eta$ stands for learning rate and $N(i, x)$ is a function that defines neighborhood. Classical Self Organizing Map (SOM) can be created when function $N(i, x)$ is defined as:

$$
N(i, x)=\left\{\begin{array}{lll}
1 & \text { for } & d(i, w) \leqslant \lambda \\
0 & \text { for } \text { others }
\end{array}\right\}
$$

where $d(i, w)$ is euclidean distance between winning and $i$-th neuron. $\lambda$ is neighborhood radius. To train Kohonen SOM euclidean distance between input vector and all neural weights has to be calculated. Neuron that has the shortest distance to input vector (the winner) is chosen and it's weights are slightly modified to direction represented by input vector. Then neighboring neurons are taken and their weights are modified in the same direction. $\eta$ and $\lambda$ are multiplied with $\Delta \eta$ and lambda $\Delta \lambda$ respectively during each learning iteration. These two last parameters are always less that one. Therefore, $\eta$ and $\lambda$ become smaller during learning process. At the beginning SOM tries to organize itself globally and with following iterations it performs more and more local organization, because learning rate and neighborhood get smaller. 


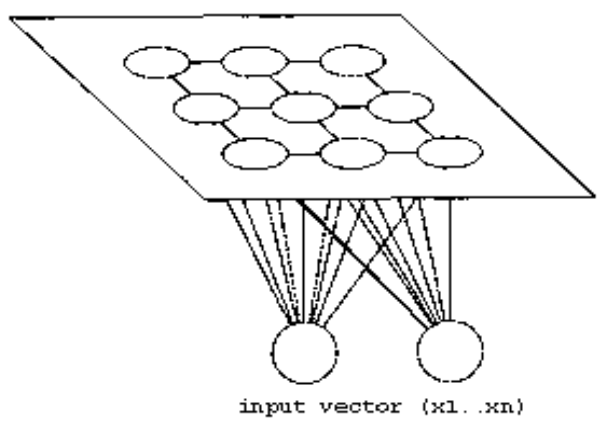

Figure 1: Kohonen SOM with two dimensional neighborhood and input vector.

Kohonen SOM is shown in Figure 1. It maps input vectors of any dimension onto map with one, two or more dimensions. Input patterns, which are similar to one another in the input space are put close to one another in the map. The input vector is passed to every neuron. A Kohonen SOM is made of a vector or matrix of output neurons. If vector representation is chosen each neuron has two neighbors (on the left and on the right). It is called one dimensional neighborhood:

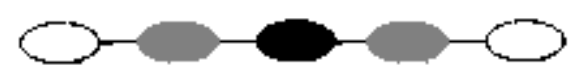

Figure 2: One dimensional neighborhood of Kohonen SOM

If two dimensional matrix representation is used, neurons have 4 neighbors (left, right, top and bottom). This is classical two dimensional neighborhood:

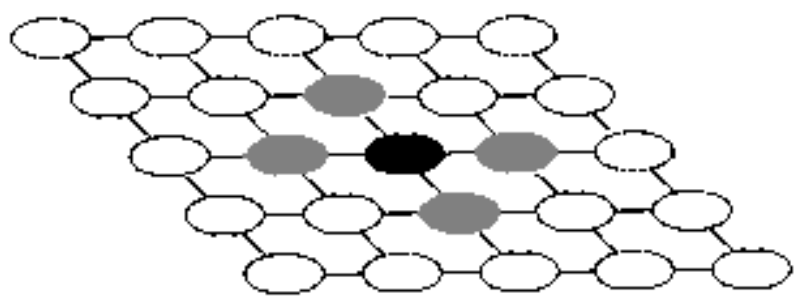

Figure 3: Classical two dimensional neighborhood

Neighborhood can be expanded. Instead of taking four nearest neuron's, 8 or more can be taken:

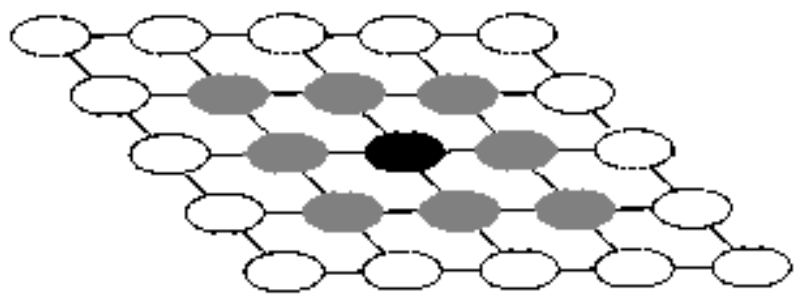

Figure 4: Extended two dimensional neighborhood of Kohonen SOM

As many dimensions can be used as needed: 1D, 2D, 3D or more. However, 2D neighborhood is most common.

Basic SOM algorithm can be described as follows:

\section{procedure train_SOM}

begin

randomize weights for all neurons

for ( $i=1$ to iteration_number) do

begin

take one random input patter

find the winning neuron

find neighbors of the winner

modify synaptic weights of these neurons

reduce the $\eta$ and $\lambda$ 
end

\section{Experiments on self-organization}

Most interesting results of self-organization can be achieved in networks that have two dimensional input vector and two dimensional neighborhood. In this case input to network consists of two values: $x$ and $y$, which represent a point in two dimensional space. This kind of network can map two dimensional objects in such a way that a mesh which covers this object is created. This process is illustrated in Figure 5. Each example consists of six squares. First one shows object that should be learned. Second square illustrates network just after randomization of all neural weights. Following squares describe learning process. Please note that each neuron (a circle) represents a point whose coordinates are equal to neuron's weights.
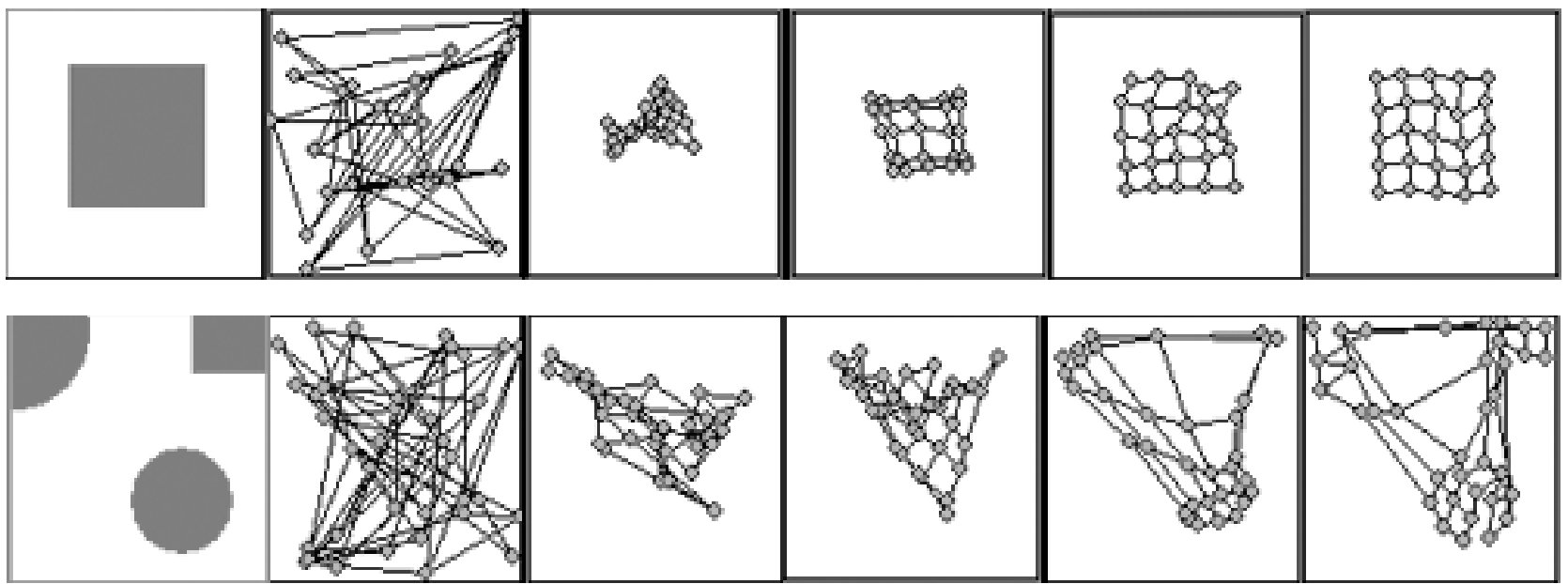

Figure 5: Self-organization of a network with two dimensional neighborhood.

These figures illustrate that Kohonen neural network is a powerful self-organizing and clustering tool. However, it is also possible to create a network with one dimensional neighborhood and two dimensional input. Learning process of this is shown in Figure 6.
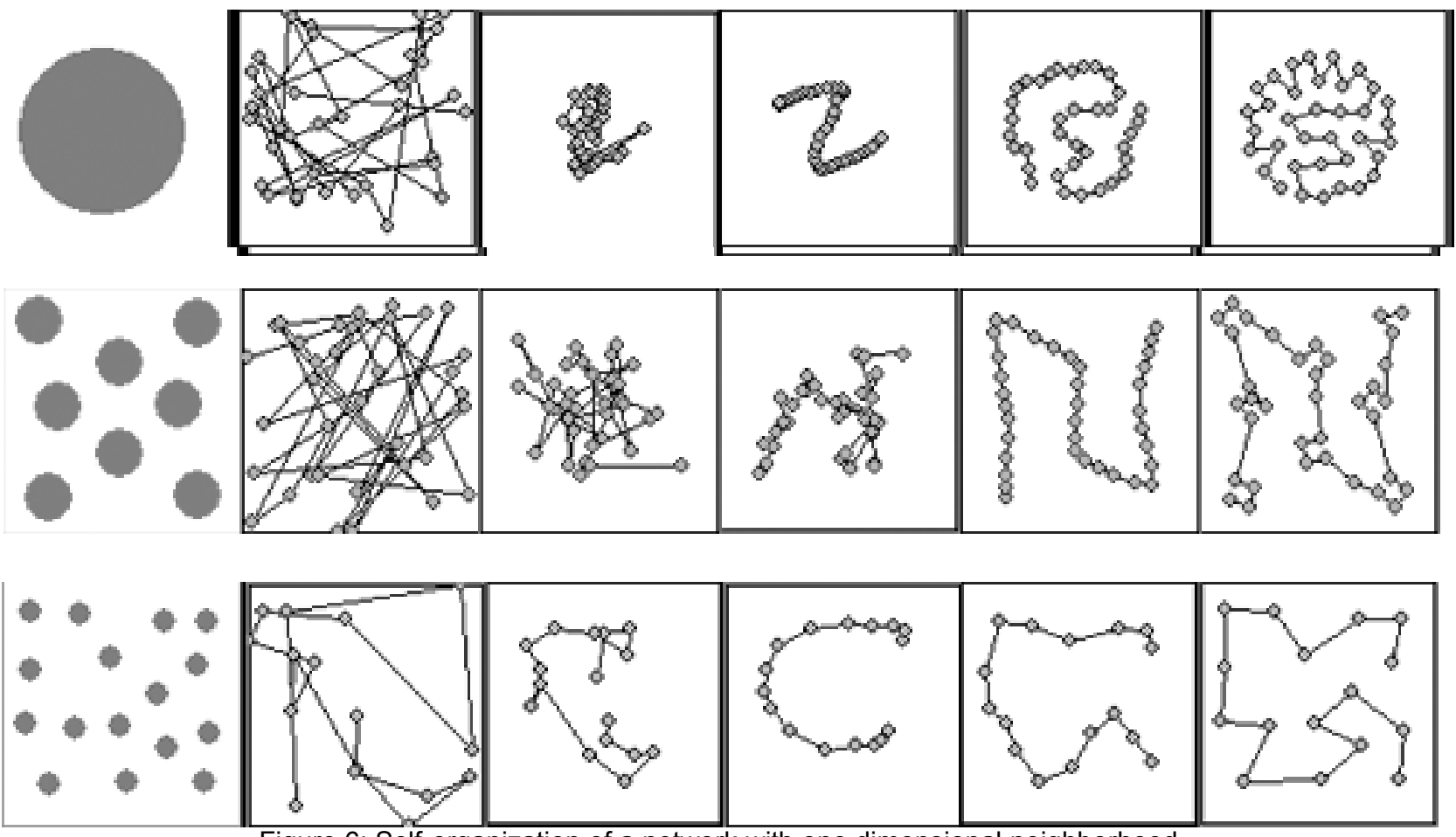

Figure 6: Self-organization of a network with one dimensional neighborhood. 
It can be observed that this network tries to organize it's neurons in such a way, that a relatively short route between all neurons emerges. These experiments were a stimulus to build a system based on Kohonen one dimensional SOM that would solve TSP problems.

\section{SOM based TSP solver}

To solve TSP problem a one dimensional network must be created. Number of neurons must be equal to the number of cities. If the weights of a neuron are equal to some city's coordinates this neuron represents that city. In other words a neuron and a city are assigned to each other. All neurons are organized in a vector. This vector represents sequence of cities that must be visited. Unfortunately, Kohonen SOM without some modifications is unable to solve TSP. The reason for this is that if neural weights are taken as coordinates of cities, they may not equal the coordinates of cities that were given. To solve the problem an algorithm that would modify Kohonen solution to one that is valid has been created. Positions of cities and positions of neurons may not equal. However, adequate neural weights and cities' coordinates are very close to each other. An algorithm that modifies neural weights so they equal to cities' coordinates has been applied. If neuron $A$ is assigned to a city $B$ it means that weights of neuron $A$ are equal to coordinates of city $B$.

Scheme repair algorithm:

\section{procedure repair}

begin

Iterate through all neurons

begin

nearest_city $=$ find the nearest city to current neuron

if (nearest_city is not assigned to any neuron)

else

assign nearest_city and current neuron

end

delete this neuron

Iterate through all cities

begin

if (current city is not assigned to any neuron)

begin

create a new_neuron and assign it to current city

nearest_neuron $=$ find the nearest neuron to current city

insert new_neuron before or after nearest_neuron, depending on which tour is locally

end

end

end

This simple algorithm gives quite good results and is fast. However, its solution is not locally optimal. Therefore, it is optimized using well know 2-opt algorithm. In this case 2-opt works fast even for large amount of cities, because current solution is already good. Usually 2-opt does not change the solution a lot (Figure 9). The 2-opt algorithm is based on a simple rule. It selects a part of the tour, reverses it, and inserts back in the cycle. If the new tour is shorter than the original cycle, then it is replaced. The algorithm stops when no improvement can be done. For example if there is a cycle $(A, B, C, D, E, F)$ and a path $(B, C, D)$ is reversed, then the new cycle is: $(A, D, C, B, E, F)$. After 2-opt optimization the solution is locally optimal. 

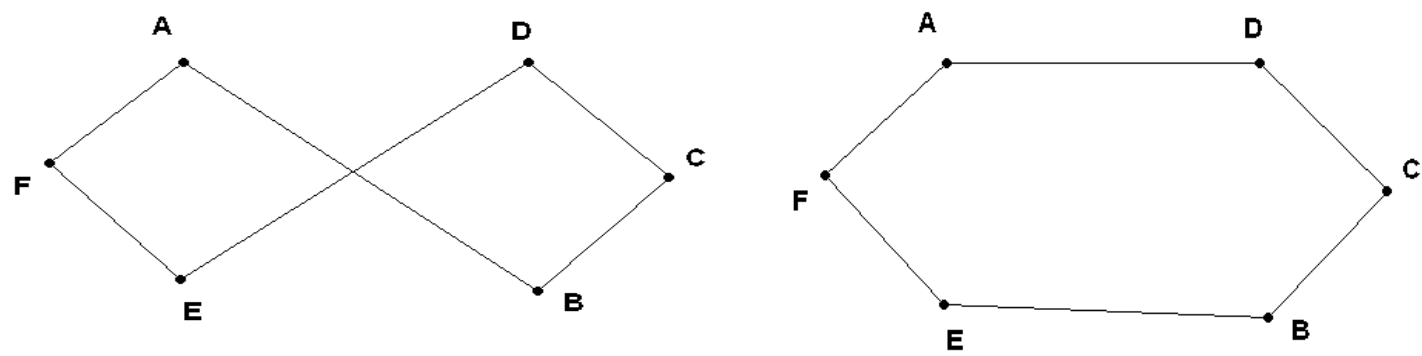

Figure 8: 2-opt optimization. If there is a cycle $(A, B, C, D, E, F)$ and a path $(B, C, D)$ is reversed, then the new cycle is: $(A, D, C, B, E, F)$
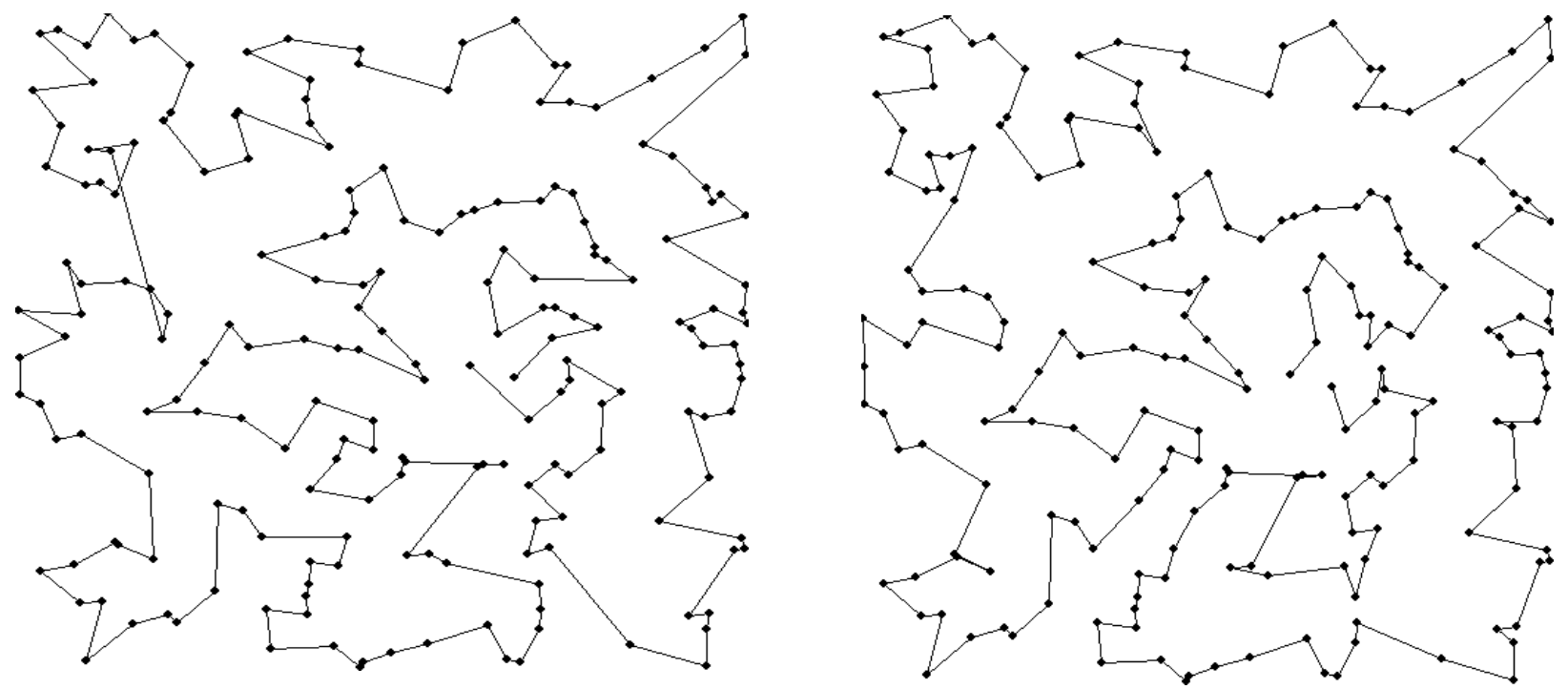

Figure 9: SOM solution without 2-opt optimization (left). There are two local loops on the left. First and last neuron can be seen in the middle. They are not connected it the picture, but distance between them is also computed. The same solution improved by 2-opt (right). Loops on the left have been erased. Additional changes can be observed.

Optimal Kohonen SOM training parameters should be chosen adequately to number of cities. It was found empirically that good training parameters are:

- for 100 cities:

$$
\begin{aligned}
& \eta=0.6 \\
& \Delta \eta=0.9997 \\
& \Delta \lambda=0.999
\end{aligned}
$$

- for 500 cities:

$$
\begin{aligned}
& \eta=0.7 \\
& \Delta \eta=0.999985 \\
& \Delta \lambda=0.9994
\end{aligned}
$$

- for 1000 cities:

$$
\begin{aligned}
& \eta=0.9 \\
& \Delta \eta=0.99992 \\
& \Delta \lambda=0.9996
\end{aligned}
$$

In each case number of iterations was set to 25000 . 


\section{The Experiment}

How good is this hybrid solver? There are two main ways to test TSP solutions:

- Using city sets taken from TSPLIB. Some optimal solutions are already there.

- Using randomly chosen cities.

TSPLIB city sets are rather hard to solve. The reason for this is that in many cases cities are not chosen randomly (Figure 10). Often larger city sets consist of smaller patterns. City set shown in Figure 11 consist of two different patterns, but each of them is used eight times. Therefore, the optimal tour is identical in each one of these smaller patterns (Figure 10, left). SOM tries to figure out a unique tour in each smaller pattern (Figure 10, right).
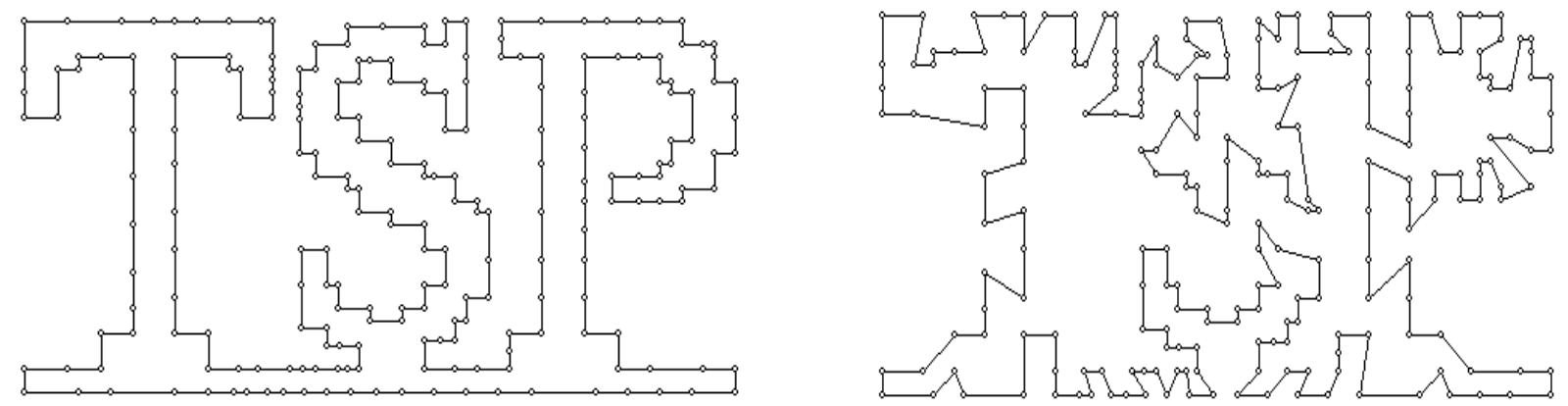

Figure 10: Optimal tour for 225 city set taken from TSPLIB (left). It's length is 3916.

Tour generated by SOM 2-opt hybrid (right). It's length is 4130 , which is $5.19 \%$ worse than optimum.
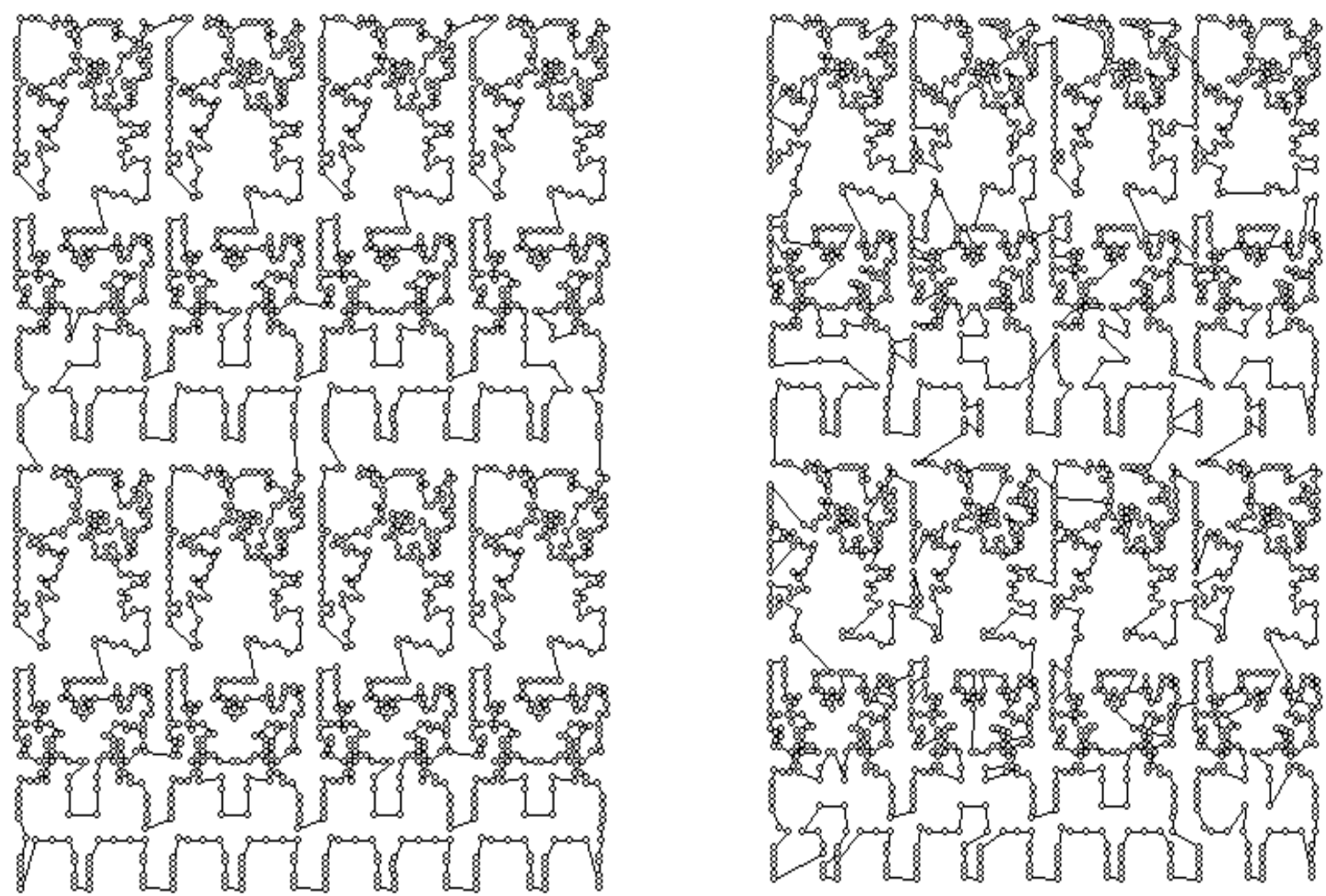

Figure 11: Optimal tour for 2392 city set taken from TSPLIB (left). It's length is 378037 . Tour generated by SOM 2-opt hybrid (right). It's length is 411442 , which is $8.12 \%$ worse than optimum.

Testing using randomly chosen cities is more objective. It is relied on the Held-Karp Traveling Salesman bound [8]. An empirical relation for expected tour length is used: 


$$
L=k \sqrt{n \cdot R}
$$

where $L$ is expected tour length, $n$ is a number of cities, $R$ is an area of square box on which cities are placed and $k$ is an empirical constant. For $n \geqslant 100$ it is:

$$
k=0.70805+\frac{0.52229}{\sqrt{n}}+\frac{1.31572}{n}-\frac{3.07474}{n \sqrt{n}}
$$

Three random city sets were used in this experiment (100, 500, 1000 cities). Square box edge length was 500.

All statistics for SOM were generated after 50 runs on each city set. When amount of iterations was adjusted to 100 average results did not change a lot. SOM can generate a tour in relatively short time. 225 city set is solved during just $300 \mathrm{~ms}$, and 1000 city set in less then 2.5 second (using machine described later). Average tour lengths for city sets up to 2000 cities are around 5 to 6 percent worse than optimum. SOM approach can generate solutions that are almost always less that $10 \%$ worse from the optimal tour. However, in most cases the difference is just a few percent. SOM has been compared with evolutionary algorithm (EA). EA used Enhanced Edge Recombination (EER) operator[2][5], Steady-State survivor selection (where always the worst solution is replaced), Tournament parent selection with tournament size depending on number of cities and population size. Scramble mutation was used. Optimal mutation rate depends on amount of cities and state of evolution. Therefore, self-adapting mutation rate has been used. Every genotype has it's own mutation rate, which is modified in a similar way as in Evolution Strategies. This strategy adapts mutation rate to number of cities and evolution state automatically, so it's not needed to check manually which parameters are optimal for each city set. Evolution stops when population converges. Population size was set to 1000 (as in [2]). With smaller populations EA did not work that well. When EA stopped it's best solution was optimized by 2opt algorithm. Results for both SOM and EA are shown in table below. All statistics for SOM were generated after 50 runs on each city set. For EA there were 10 runs of the algorithm for sets: EIL51, EIL101 and RAND100. For other sets EA was run only once. Optimum solutions for instances taken from TSPLIB were already there and optimum solutions for random instances are calculated from empirical relation described above. All computations were performed on AMD Athlon 64-bit 3500+ processor. However, the program was built using 32-bit compiler, so it did not use full computational power of this machine. Tournament size was 1.3 and population size was 1000 .

\begin{tabular}{|l|r|r|r|r|r|r|r|}
\hline \multicolumn{1}{|c|}{} & \multicolumn{3}{|c|}{ Self-Organizing Map } & \multicolumn{3}{c|}{ Evolutionary Algorithm } \\
\hline Instances & \multicolumn{1}{|c|}{ Optimum } & Ave. Result & \multicolumn{1}{c|}{ Best Result } & Ave. Time & Ave. Result & Best Result & Ave. Time \\
\hline EIL51 & 426 & 444 & 431 & 0.068 & 428.2 & 426 & 10 \\
\hline EIL101 & 629 & 662 & 646 & 0.127 & 653.3 & 639 & 75 \\
\hline TSP225 & 3916 & 4193 & 4106 & 0.302 & ---- & 4044 & 871 \\
\hline PCB442 & 50778 & 56634 & 55138 & 0.703 & ---- & 55657 & 10395 \\
\hline PR1002 & 259045 & 278481 & 274036 & 2.425 & --- & 286908 & 25639 \\
\hline PR2392 & 378037 & 418739 & 411442 & 12.965 & --- & --- & ---- \\
\hline RAND100 & 3851,81 & 4051 & 3883 & 0.131 & 3931.4 & 3822 & 69.6 \\
\hline RAND500 & 8203,73 & 8888 & 8697 & 0.824 & --- & 9261 & 11145 \\
\hline RAND1000 & 11475,66 & 12483 & 12343 & 2.311 & --- & 12858 & 56456 \\
\hline
\end{tabular}

Experiments show that EA finds better solutions for instances with up to 101 cities. Both Average Result and Best Result are better that SOM. For city sets with 50 or less cities EA finds optimum in almost every execution. Results for 225 cities are comparable for both algorithms, however for larger amount of cities (442 and more) SOM wins the competition. The more cities the instance has, the bigger the difference between both algorithms. With more cities search space increases significantly and EA needs bigger population. For TSP225 with population size 1000 EA's result was 4044, but when population size was expanded to 3000 a tour with length 3949 was found - much better than SOM's solution. This underlines the fact that when EA is used one can always expand population size so the algorithm has greater chance of achieving good result. Unfortunately, the algorithm is much slower then.

It is interesting to compare SOM algorithm to other non-evolutionary approach. One of the best TSP algorithms, which is also extremely fast, is Lin-Kerninghan. Results for this algorithm have been taken from [4]. The algorithm was run 10 times on each city set. Average results and average times were taken from [4] and are shown below. A Pentium Pro $180 \mathrm{Mhz}$ was used, so it is rather hard to compare times from this 
table and times for SOM (a 3.5 Ghz processor was used). Anyway, Lin-Kerninghan is faster than SOM even on a many times slower machine. There is not a big difference in time for a small 51-city instance $(0.012$ seconds for Lin-Kerninghan and 0.068 seconds for SOM). On the other hand for 2392-city instance LinKerninghan needed just 0.719 seconds and SOM almost 13 seconds. This is because SOM is optimized by 2opt, which is the slowest part of this algorithm. When average results are compared it can be easily seen that Lin-Kerninghan wins in all cases. The more cities there is, the bigger the difference between both algorithms.

\begin{tabular}{|l|r|r|r|}
\hline & & \multicolumn{2}{|c|}{ Lin-Kerninghan } \\
\hline Instances & \multicolumn{1}{|c|}{ Optimum } & Ave. Result & \multicolumn{1}{c|}{ Ave. Time } \\
\hline ElL51 & 426 & 427.4 & 0.012 \\
\hline ElL101 & 629 & 640 & 0.039 \\
\hline PCB442 & 50778 & 51776.5 & 0.137 \\
\hline PR2392 & 378037 & 389413 & 0.719 \\
\hline
\end{tabular}

SOM was also used to generates initial population for EA. Such initialization takes only a fraction of time needed for EA to finish, because SOM is a fast algorithm. In this case EA tended to converge much faster and finally it did not improve much best solution generated by SOM alone. It seems that all initial solutions were very similar to each other, thus population diversity was low, so EA lost exploration abilities.

\section{Conclusions}

It seems that SOM-2opt hybrid is not a very powerful algorithm for the TSP. It has been outperformed by both: EA and Lin-Kerninghan algorithms. It's speed might be impressive, but it still is slower than LinKerninghan.

There are a couple of things that can be optimized. Here are some of them:

- $\quad$ an optimal network parameter settings should be found ( $\eta, \Delta \eta, \Delta \lambda$, number of iterations)

- experiments with other self-organizing networks should be performed, Gaussian neighborhood and "conscience mechanism" may be applied. Conscience mechanism can improve TSP solutions generated by neural networks, as reported in [6].

- 2 opt algorithm is not very sophisticated. Some other optimization method may be better.

The are many algorithms that solve permutation problems. Evolutionary Algorithms have many different operators that work with permutations. EER is one of the best operators for the TSP [5]. However, it was proved that other permutation operators, which are worse for the TSP than EER, are actually better for other permutation problems (like warehouse/shipping scheduling) [5]. Therefore, it might be possible that SOM 2opt hybrid might work better for other permutation problems than for TSP.

\section{Acknowledgements}

I am grateful to Prof. Zbigniew Michalewicz for influencing and helping me to write this paper.

\section{References}

1. Kohonen T. (2001), Self-Organizing Maps, Springer, Berlin

2. Michalewicz Z. (1996), Genetic Algorithms + Data Structures = Evolution Programs, Springer - Verlag

3. Arbib M. (1998), The Handbook of Brain Theory and Neural Networks, The MIT Press

4. Tao G., Michalewicz Z., Inver-over Operator for the TSP

5. Starkweather T., McDaniel S., Whitley C., Mathias K., Whitley D., (1991), A Comparison of Genetic Sequencing Operators

6. Burke Laura I., (1993), Neural Methods for the Traveling Salesman Problem: Insights From Operations Research

7. Xu W., Tsai W. T. (1990), Effective Neural Algorithm for the Traveling Salesman Problem

8. Johnson, D.S., McGeoch, L.A., and Rothberg, E.E., Asymptotic experimental analysis for the Held-Karp traveling salesman bound 
9. Lin S., Kernighan B. W., (1971), An effective Heuristic for the Traveling Salesman Problem

10. Reinelt G., (1995), TSPLIB 95 documentation, University of Heidelberg

11. Tadeusiewicz R., (1993), Sieci Neuronowe, Akademicka Oficyna Wydawnicza

12. Osowski S., (1996), Sieci Neuronowe w ujęciu algorytmicznym, WNT

13. Duch W., Korbowicz J., Rutkowski L., Tadeusiewicz R., (2000), Biocybernetyka i Inżynieria Biomedyczna: Sieci Neuronowe, Akademicka Oficyna Wydawnicza 